Volume 71, Number 1, Pages 51-67 (2022)

DOI:10.31801/cfsuasmas.946910

ISSN 1303-5991 E-ISSN 2618-6470

Research Article; Received:June 2, 2021; Accepted: July 17, 2021

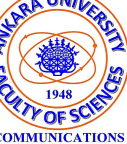

\title{
A NUMERICAL METHOD ON BAKHVALOV SHISHKIN MESH FOR VOLTERRA INTEGRO-DIFFERENTIAL EQUATIONS WITH A BOUNDARY LAYER
}

\author{
Hayriye GUCKIR CAKIR, ${ }^{1}$ Firat $\mathrm{CAKIR}^{2}{ }^{2}$ and Musa $\mathrm{CAKIR}^{3}$ \\ ${ }^{1}$ Department of Mathematics, Adıyaman University, Adıyaman, TURKEY \\ ${ }^{2}$ Department of Mathematics, Batman University, Batman, TURKEY \\ ${ }^{3}$ Department of Mathematics, Van Yüzüncü Yıl University, Van, TURKEY
}

\begin{abstract}
We construct a finite difference scheme for a first-order linear singularly perturbed Volterra integro-differential equation (SPVIDE) on Bakhvalov-Shishkin mesh. For the discretization of the problem, we use the integral identities and deal with the emerging integrals terms with interpolating quadrature rules which also yields remaining terms. The stability bound and the error estimates of the approximate solution are established. Further, we demonstrate that the scheme on Bakhvalov-Shishkin mesh is $O\left(N^{-1}\right)$ uniformly convergent, where $N$ is the mesh parameter. The numerical results are also provided for a couple of examples.
\end{abstract}

\section{INTRODUCTION}

In this present work, we are specifically consider the following class of the singularly perturbed linear Volterra integro-differential equations (SPVIDEs)

$$
L u:=\varepsilon u^{\prime}+a(x) u+\lambda \int_{0}^{x} K(x, t) u(t) d t=f(x), \quad x \in I=[0, \ell],
$$

subject to

$$
u(0)=A,
$$

where $0<\varepsilon \ll 1$ is a small perturbation parameter. We assume $a(x) \geq \alpha>0$, $f(x)(x \in I)$ and $K(x, t)((x, t) \in I \times I)$ are sufficiently smooth functions such

2020 Mathematics Subject Classification. 65L11, 65L12, 65L20, 65R20.

Keywords. Singularly perturbed, VIDE, difference schemes, uniform convergence, error estimates.

■hayriyeguckir@gmail.com-Corresponding author; firat.cakir@hotmail.com; cakirmusa@msu.edu

(D) 0000-0002-1570-1534; 0000-0001-5555-4244; 0000-0002-1979-570X. 
that the initial layer for the solution $u(x)$ occurs at $x=0$ for small values of $\varepsilon$. Volterra integro-differential equations (VIDEs) are an important class of equations which are extensively used to model many sciencitific problems such as population dynamics 13|, filament streching 5 and epidemics 37 . Many techniques have been introduced to solve VIDEs analytically. The variational iteration method, the Adomian decomposition method and the homotopy perturba-tion method are some well-known analytical methods to solve $\operatorname{VIDEs}(40|, 9,17|)$. Recently, a new approach on the variational analytical method has been introduced to solve Volterra-Fredholm Integral equations which does not require construction of the variational principle 18 . Further, a finite difference scheme is utilized to examine the numerical solutions of a non-linear VIDE in 11.

Singularly perturbed differential equations, which have the highest order derivative term multi- plied with a small positive number $\varepsilon$, possess solutions with interior or boundary layers. Boundary layers are regions where rapid changes occur which makes solving such problems more challenging. Since standard schemes fail to give the accurate results for problems with boundary layer for small $\varepsilon$ values, numerical solutions of such problems have been of interest to many researchers (|12|, |15, |16, 22, 28, 29|, 31|,|34,|38, 35 ). Singularly perturbed Volterra integro-differential equations (SPVIDEs) have been widely used to model problems in many science fields such as epidemic dynamics, synchronous control systems, filament stretching and heat transfer $(|6,7| 14,,20|, 21| 32,, \mid 33$ ). A review on the literature of the SPVIDEs was given in 25 . Further, asymptotic expansions derivation of the solutions to SPVIDEs are studied in $|6|, 7||$,25 . In $\mid 32$ a problem of nonlinear SPVIDE modelling the elongation ratio of filament is studied and the qualitative properties of the solution is discussed under some physically interesting assumptions. In $\mid 5$, a specific integro-differential equation with a boundary layer which describes filament stretching process is considered and the leading order behavior of the problem is examined by an asymptotic method. Singularly perturbed integro differential equations have been also an interest to many researchers. In 23 and 24 , the numerical solutions of singularly perturbed integrodifferential and integro-differential-algebraic equations are analyzed by the implicit Runge-Kutta methods. An exponential finite difference method is applied for the inner and outer layers and a type of implicit Runge-Kutta method is performed to obtain the outer layer solutions of SPVIDEs in 36. A finite Legendre expansion is constructed to solve different kinds of integral equations and integro-differential equations $\mid 26$. In $\mid 19$, tension spline collocation methods are utilized to numerically discretize singularly perturbed Volterra integral and integro-differential equations. In $\mid 39$, the authors present different types of exponential schemes to solve SPVIDEs and the stability analysis of the schemes is examined. Fitted difference schemes are also proven to provide accurate results in the solution process of different types of singularly perturbed problems. In $|2|$, an exponentially fitted difference method is designed on a uniform mesh to solve linear SPVIDEs. First-order convergent 
finite difference schemes are developed to solve linear first order SPVIDEs with delay in $4, \mid 27$. In $|3|$, using a fitted difference operator a second-order difference scheme is constructed on a piecewise uniform mesh to solve linear SPVIDEs.

In this present work, we mainly construct a uniform convergent difference scheme on a Bakhvalov-Shishkin mesh for the problem (1)-(2). Bakhvalov-Shishkin mesh is a mixed version of the Shishkin mesh and Bakhvalov mesh which are known to yield accurate results for singularly perturbed problems with boundary layers. In 30 , the author demonstrated that the results from an upwind difference scheme on Bakhvalov-Shishkin mesh applied to a linear convection-diffusion equation are more accurate than the results from the upwind scheme on a Shishkin mesh. Further, a finite difference scheme on Bakhvalov-Shishkin mesh is utilized to deal with a singularly perturbed boundary value problem in 10 .

The rest of the paper is organized in the following order. In Section 2, the asymptotic estimates on the exact solution to (1)-(2) are established. In Section 3 , we define the Bakhvalov-Shishkin mesh points according to the boundary layer conditions of the problem (1)-(2) and derive a finite difference scheme utilizing the integral identities with exponential basis functions and then applying interpolating quadrature rules provided in 1 to the integral terms. In Section 4, we establish the stability bounds and the error estimates of the numerical solution and as a result we show that the scheme demonstrates $O\left(N^{-1}\right)$ uniform convergence with respect to the perturbation parameter. We also provide the numerical results in Section 5 .

\section{Asymptotic Behavior of the Solution}

In the following lemma, we establish a priori estimates for the asymptotic behavior of the solution to the problem (1)-(2).

Lemma 1. Let $a, f \in C(I)$ and $K \in C(I \times I)$. The solution $u$ to the problem (1)-(2) holds

$$
\|u\|_{\infty} \leq C
$$

where

$$
C=\left(A+\alpha^{-1}\|f\|_{\infty}\right) e^{\lambda \bar{K} \alpha^{-1} \ell},
$$

and $\bar{K}=\max _{I \times I}|K(x, t)|$. In addition, if $a, f \in C^{1}(I)$ and $K \in C^{1}(I \times I)$ with

$$
\left|\frac{\partial}{\partial x} K(x, t)\right| \leq \bar{K}_{1}<\infty
$$

then the solution $u(x)$ satisfies

$$
\left|u^{\prime}(x)\right| \leq C\left(1+\frac{1}{\varepsilon} e^{-\frac{\alpha x}{\varepsilon}}\right), x \in I .
$$

Proof. To establish the first estimate given in (3) we start by rewriting (1) as

$$
\varepsilon u^{\prime}+a(x) u=F(x),
$$


where

$$
F(x)=f(x)-\lambda \int_{0}^{x} K(x, t) u(t) d t .
$$

Solving the equation (6) with $u(0)=A$ yields

$$
u(x)=A e^{-\frac{1}{\varepsilon} \int_{0}^{x} a(s) d s}+\frac{1}{\varepsilon} \int_{0}^{x} F(\xi) e^{-\frac{1}{\varepsilon} \int_{\xi}^{x} a(s) d s} d \xi,
$$

and further we calculate

$$
|u(x)| \leq|A| e^{-\frac{1}{\varepsilon} \int_{0}^{x} a(s) d s}+\frac{1}{\varepsilon} \int_{0}^{x}|F(\xi)| e^{-\frac{1}{\varepsilon} \int_{\xi}^{x} a(s) d s} d \xi .
$$

Since we have $a(x) \geq \alpha>0$, it follows

$$
\begin{aligned}
|u(x)| & \leq|A| e^{-\frac{1}{\varepsilon} \int_{0}^{x} \alpha d s}+\frac{1}{\varepsilon} \int_{0}^{x}|F(\xi)| e^{-\frac{1}{\varepsilon} \int_{\xi}^{x} \alpha d s} d \xi \\
& =|A| e^{-\frac{\alpha x}{\varepsilon}}+\frac{1}{\varepsilon} \int_{0}^{x}|F(\xi)| e^{-\frac{\alpha(x-\xi)}{\varepsilon}} d \xi .
\end{aligned}
$$

Here, by the definition of $F(x)$ in (7), we get

$$
|F(x)| \leq\|f\|_{\infty}+\lambda \bar{K} \int_{0}^{x}|u(t)| d t .
$$

Substituting (9) into (8) yields

$$
\begin{aligned}
|u(x)| & \leq|A| e^{-\frac{\alpha x}{\varepsilon}}+\frac{1}{\varepsilon} \int_{0}^{x}\left(\|f\|_{\infty}+\lambda \bar{K} \int_{0}^{\xi}|u(t)| d t\right) e^{-\frac{\alpha(x-\xi)}{\varepsilon}} d \xi \\
& =|A| e^{-\frac{\alpha x}{\varepsilon}}+\frac{1}{\varepsilon}\|f\|_{\infty} \int_{0}^{x} e^{-\frac{\alpha(x-\xi)}{\varepsilon}} d \xi+\frac{\lambda \bar{K}}{\varepsilon} \int_{0}^{x} \int_{0}^{\xi}|u(t)| d t e^{-\frac{\alpha(x-\xi)}{\varepsilon}} d \xi
\end{aligned}
$$

We integrate by parts the last term with double integral here

$$
\begin{aligned}
|u(x)| & \leq|A| e^{-\frac{\alpha x}{\varepsilon}}+\alpha^{-1}\|f\|_{\infty}\left(1-e^{-\frac{\alpha x}{\varepsilon}}\right)+\alpha^{-1} \lambda \bar{K}\left(1-e^{-\frac{\alpha x}{\varepsilon}}\right) \int_{0}^{x}|u(t)| d t \\
& \leq|A|+\alpha^{-1}\|f\|_{\infty}+\alpha^{-1} \lambda \bar{K} \int_{0}^{x}|u(t)| d t .
\end{aligned}
$$


An application of the Gronwall's inequality to (10) provides

$$
\begin{aligned}
|u(x)| & \leq\left(|A|+\alpha^{-1}\|f\|_{\infty}\right) e^{\alpha^{-1} \lambda \bar{K} x} \\
& \leq\left(|A|+\alpha^{-1}\|f\|_{\infty}\right) e^{\alpha^{-1} \lambda \bar{K} \ell},
\end{aligned}
$$

which leads to the desired result in (3).

For the next estimate provided in (5), we first differentiate the equation (1) and have

$$
\varepsilon u^{\prime \prime}+a^{\prime}(x) u+a(x) u^{\prime}+\lambda K(x, x) u+\lambda \int_{0}^{x} \frac{\partial}{\partial x} K(x, t) u(t) d t=f^{\prime}(x) .
$$

Then, letting

$$
v(x)=u^{\prime}(x)
$$

and

$$
g(x)=f^{\prime}(x)-a^{\prime}(x) u-\lambda K(x, x) u-\lambda \int_{0}^{x} \frac{\partial}{\partial x} K(x, t) u(t) d t,
$$

we have

$$
\varepsilon v^{\prime}+a(x) v=g(x) .
$$

In a similar manner to the previous work above, we solve (12)

$$
v(x)=v(0) e^{-\frac{1}{\varepsilon} \int_{0}^{x} a(s) d s}+\frac{1}{\varepsilon} \int_{0}^{x} g(\xi) e^{-\frac{1}{\varepsilon} \int_{\xi}^{x} a(s) d s} d \xi .
$$

Then, we have

$$
\begin{aligned}
|v(x)| & \leq|v(0)| e^{-\frac{1}{\varepsilon} \int_{0}^{x} a(s) d s}+\frac{1}{\varepsilon} \int_{0}^{x}|g(\xi)| e^{-\frac{1}{\varepsilon} \int_{\xi}^{x} a(s) d s} d \xi \\
& \leq|v(0)| e^{-\frac{1}{\varepsilon} \int_{0}^{x} \alpha d s}+\frac{1}{\varepsilon} \int_{0}^{x}|g(\xi)| e^{-\frac{1}{\varepsilon} \int_{\xi}^{x} \alpha d s} d \xi \\
& \leq|v(0)| e^{-\frac{\alpha x}{\varepsilon}}+\frac{1}{\varepsilon} \int_{0}^{x}|g(\xi)| e^{-\frac{\alpha(x-\xi)}{\varepsilon}} d \xi .
\end{aligned}
$$


Here, by the formula of $g(x)$ given in (11), from (3) and knowing that $a, f \in C^{1}(I)$, $K \in C^{1}(I \times I)$ and from (4) we obtain

$$
\begin{aligned}
|g(x)| & \leq\left\|f^{\prime}\right\|_{\infty}+\left\|a^{\prime}\right\|_{\infty}|u|+\lambda \bar{K}|u|+\lambda \bar{K}_{1} \int_{0}^{x}|u(t)| d t \\
& \leq\left\|f^{\prime}\right\|_{\infty}+C\left(\left\|a^{\prime}\right\|_{\infty}+\lambda \bar{K}_{1}+\ell\right),
\end{aligned}
$$

which implies $\|g\|_{\infty} \leq C_{*}$ for a $C_{*} \in \mathbb{R}$. Hence, utilizing this estimate on $g(x)$ in (13) provides

$$
\begin{aligned}
|v(x)| & \leq|v(0)| e^{-\frac{\alpha x}{\varepsilon}}+\frac{1}{\varepsilon}\|g\|_{\infty} \int_{0}^{x} e^{-\frac{\alpha(x-\xi)}{\varepsilon}} d \xi \\
& \leq|v(0)| e^{-\frac{\alpha x}{\varepsilon}}+\alpha^{-1} C_{*}\left(1-e^{-\frac{\alpha x}{\varepsilon}}\right) .
\end{aligned}
$$

On the other hand, inserting $x=0$ in (1) and since $a, f \in C^{1}(I)$ it follows that

$$
|v(0)|=\left|u^{\prime}(0)\right|=\frac{1}{\varepsilon}|f(0)-A a(0)| \leq \frac{c}{\varepsilon} .
$$

Substituting this into (15) yields

$$
|v(x)| \leq \frac{c}{\varepsilon} e^{-\frac{\alpha x}{\varepsilon}}+\alpha^{-1} C_{*}\left(1-e^{-\frac{\alpha x}{\varepsilon}}\right),
$$

which provides the desired result.

\section{Difference Scheme}

3.1. Notation. Before we proceed to the definition of the mesh points and discretization of the problem we provide the notation we use throughout the paper. Let $\bar{\omega}_{h}=\left\{0=x_{0}<x_{1}<x_{2}<\cdots<x_{N-1}<x_{N}=\ell\right\}$ denote a non-uniform mesh on $[0, \ell]$. For each $i=0, \cdots, N$, let $h_{i}=x_{i}-x_{i-1}$ denote the step size. For any continuous mesh function $v_{i}$ defined on $\omega_{h}$ we use the notation

$$
v_{\bar{x}, i}=\frac{v_{i}-v_{i-1}}{h_{i}}
$$

for backward difference.

3.2. Discretization. In this section, we construct our difference scheme based on Bakhvalov-Shishkin mesh. According to this mesh construction, we divide the domain into two subintervals $[0, \sigma]$ and $[\sigma, \ell]$, where $\sigma$ is the transition parameter. For a positive even discretization parameter $N$, we determine the transition parameter $\sigma$ as

$$
\sigma=\min \left\{\frac{\ell}{2}, \varepsilon \alpha^{-1} \ln N\right\} .
$$


We assume $\varepsilon \ll N^{-1}$ as it is used in practice. We define a set of mesh points as the following

$$
x_{i}= \begin{cases}-\alpha^{-1} \varepsilon \ln \left[1-2\left(1-N^{-1}\right) \frac{i}{N}\right], & x_{i} \in[0, \sigma], i=0,1, \cdots, \frac{N}{2}, \\ \sigma+\left(i-\frac{N}{2}\right) h, \quad h=\frac{2(\ell-\sigma)}{N}, & x_{i} \in[\sigma, \ell], i=\frac{N}{2}+1, \cdots, N .\end{cases}
$$

To derive the difference approximation, we use the following integral identity

$$
\chi_{i}^{-1} h_{i}^{-1} \int_{x_{i-1}}^{x_{i}} L u(x) \varphi_{i}(x) d x=\chi_{i}^{-1} h_{i}^{-1} \int_{x_{i-1}}^{x_{i}} f(x) \varphi_{i}(x) d x,
$$

with the exponential basis function

$$
\varphi_{i}(x)=e^{-\frac{a_{i}}{\varepsilon}\left(x_{i}-x\right)}, \quad i=1, \cdots, N,
$$

where

$$
\chi_{i}=h_{i}^{-1} \int_{x_{i-1}}^{x_{i}} \varphi_{i}(x) d x=\frac{1-e^{-a_{i} \rho_{i}}}{a_{i} \rho_{i}}, \quad \rho_{i}=\frac{h_{i}}{\varepsilon} .
$$

We remark that $\varphi_{i}$ solves the equation

$$
\begin{aligned}
& -\varepsilon \varphi_{i}(x)+a_{i} \varphi_{i}(x)=0, \quad x_{i-1} \leq x \leq x_{i} \\
& \varphi_{i}\left(x_{i}\right)=1 .
\end{aligned}
$$

To obtain the difference scheme from (18), we proceed by evaluating the integrals term by term applying the interpolating quadrature rules with weight functions and obtain the remainder terms as provided in 1 . In the following, we handle the differential term on the left-hand side of (18),

$$
\begin{aligned}
\chi_{i}^{-1} h_{i}^{-1} \int_{x_{i-1}}^{x_{i}}\left[\varepsilon u^{\prime}(x)+a(x) u(x)\right] \varphi_{i}(x) d x= & \chi_{i}^{-1} h_{i}^{-1} \int_{x_{i-1}}^{x_{i}}\left[\varepsilon u^{\prime}(x)+a_{i} u(x)\right] \varphi_{i}(x) d x \\
& +\chi_{i}^{-1} h_{i}^{-1} \int_{x_{i-1}}^{x_{i}}\left[a(x)-a_{i}\right] u(x) \varphi_{i}(x) d x \\
= & \varepsilon \theta_{i} u_{\bar{x}, i}+a_{i} u_{i}+R_{i}^{(1)},
\end{aligned}
$$

where

and

$$
\theta_{i}=\frac{a_{i} \rho_{i} e^{-a_{i} \rho_{i}}}{1-e^{-a_{i} \rho_{i}}}
$$

$$
R_{i}^{(1)}=\chi_{i}^{-1} h_{i}^{-1} \int_{x_{i-1}}^{x_{i}}\left[a(x)-a_{i}\right] u(x) \varphi_{i}(x) d x .
$$

Further, applying the first quadrature rules provided in $|1|$ to the integral term in (18) twice we obtain

$$
\chi_{i}^{-1} h_{i}^{-1} \lambda \int_{x_{i-1}}^{x_{i}} \varphi_{i}(x) \int_{0}^{x} K(x, t) u(t) d t d x=\lambda \int_{0}^{x_{i}} K\left(x_{i}, t\right) u(t) d t+R_{i}^{(2)},
$$


where

$$
R_{i}^{(2)}=\lambda \int_{x_{i-1}}^{x_{i}} \frac{\partial}{\partial \xi}\left(\int_{0}^{\xi} K(\xi, t) u(t) d t\right)\left[T_{0}(x-\xi)-h_{i}^{-1}\left(x-x_{i-1}\right)\right] d \xi
$$

and $T_{0}(\lambda)=1$ for $\lambda \geq 0$ and $T_{0}(\lambda)=0$ for $\lambda<0$. Here, we apply the composite right-side rectangle rule to the integral term in the right-hand side of (27) and get

$$
\lambda \int_{0}^{x_{i}} K\left(x_{i}, t\right) u(t) d t=\lambda \sum_{j=1}^{i} h_{j} K\left(x_{i}, x_{j}\right) u_{j}+R_{i}^{(3)}
$$

where

$$
R_{i}^{(3)}=-\lambda \sum_{j=1}^{i} \int_{x_{j-1}}^{x_{j}}\left(\xi-x_{j-1}\right) \frac{\partial}{\partial \xi}\left(K\left(x_{i}, \xi\right) u(\xi)\right) d \xi
$$

Then, inserting (25) in (23) provides

$$
\chi_{i}^{-1} h_{i}^{-1} \lambda \int_{x_{i-1}}^{x_{i}} \varphi_{i}(x) \int_{0}^{x} K(x, t) u(t) d t d x=\lambda \sum_{j=1}^{i} h_{j} K\left(x_{i}, x_{j}\right) u_{j}+R_{i}^{(2)}+R_{i}^{(3)} .
$$

On the other hand, the right-hand side of (18) gets the in the form

$$
\chi_{i}^{-1} h_{i}^{-1} \int_{x_{i-1}}^{x_{i}} f(x) \varphi_{i}(x) d x=f_{i}+R_{i}^{(4)}
$$

where

$$
R_{i}^{(4)}=\chi_{i}^{-1} h_{i}^{-1} \int_{x_{i-1}}^{x_{i}}\left[f(x)-f\left(x_{i}\right)\right] \varphi_{i}(x) d x .
$$

Inserting the relations (20), (27) and (28) in (18), we obtain the difference problem for the problem (1)-(2) as

$$
\begin{aligned}
& \varepsilon \theta_{i} u_{\bar{x}, i}+a_{i} u_{i}+\lambda \sum_{j=1}^{i} h_{j} K\left(x_{i}, x_{j}\right) u_{j}=f_{i}-R_{i}, \quad i=1,2, \cdots, N \\
& u_{0}=A
\end{aligned}
$$

where

$$
R_{i}=R_{i}^{(1)}+R_{i}^{(2)}+R_{i}^{(3)}-R_{i}^{(4)} .
$$

As a result, neglecting the error term $R_{i}$ in (30) provides the following difference scheme

$$
\begin{aligned}
& L_{N} y_{i}:=\varepsilon \theta_{i} y_{\bar{x}, i}+a_{i} y_{i}+\lambda \sum_{j=1}^{i} h_{j} K\left(x_{i}, x_{j}\right) y_{j}=f_{i}, \quad i=1,2, \cdots, N, \\
& y_{0}=A,
\end{aligned}
$$

where $\theta_{i}$ defined by (21). 


\section{Stability, Error Estimates and Convergence Results}

Here, we establish the stability bound and the error estimates of the approximate solution $y$. Further, the convergence of the difference scheme provided in (32)-(33) is analyzed.

Lemma 2. Assume that $\left|F_{i}\right| \leq \mathcal{F}_{i}$ and $\mathcal{F}_{i}$ be a non-decreasing function. The solution to the problem

$$
\begin{gathered}
\ell_{N} v_{i}:=\varepsilon \theta_{i} v_{\bar{x}, i}+a_{i} v_{i}=F_{i}, \quad 1 \leq i \leq N, \\
v_{0}=A . \\
\left|v_{i}\right| \leq|A|+\alpha^{-1} \mathcal{F}_{i}, \quad 1 \leq i \leq N .
\end{gathered}
$$

Proof. The proof follows from the maximum principle for difference operators. Details can be found in 27 .

Lemma 3. Let $y_{i}$ be the solution of the problem (32)-(33). Then, $y_{i}$ satisfies

$$
\|y\|_{\infty} \leq C_{0}\left(|A|+\|f\|_{\infty}\right) .
$$

Proof. The difference scheme equation given in (32) can be rewritten in the form

$$
\theta_{i} \varepsilon y_{\bar{x}, i}+a_{i} y_{i}=F_{i}
$$

where

$$
F_{i}=f_{i}-\lambda \sum_{j=1}^{i} h_{j} K\left(x_{i}, x_{j}\right) y_{j} .
$$

For $F_{i}$, we have the estimate

$$
\begin{aligned}
\left|F_{i}\right| & \leq\left|f_{i}\right|+\lambda\left|\sum_{j=1}^{i} h_{j} K\left(x_{i}, x_{j}\right) y_{j}\right| \\
& \leq\left|f_{i}\right|+\lambda \bar{K} \sum_{j=1}^{i} h_{j}\left|y_{j}\right| \\
& \leq\|f\|_{\infty}+\lambda \bar{K} \sum_{j=1}^{i} h_{j}\left|y_{j}\right| .
\end{aligned}
$$

Then, applying Lemma 2 to (35) and utilizing this estimate provide

$$
\left|y_{i}\right| \leq|A|+\alpha^{-1}\|f\|_{\infty}+\alpha^{-1} \lambda \bar{K} \sum_{j=1}^{i} h_{j}\left|y_{j}\right| .
$$

Further, applying the difference analogue of the Gronwall's inequality to (36) we have

$$
\left|y_{i}\right| \leq\left(|A|+\alpha^{-1}\|f\|_{\infty}\right) e^{\alpha^{-1} \lambda \bar{K} \ell}
$$

which yields the result in (34). 
The error of the difference problem is given by the solution to the problem

$$
\begin{aligned}
& L_{N} z_{i}=R_{i}, \quad 1 \leq i \leq N \\
& z_{0}=0
\end{aligned}
$$

Lemma 4. Suppose that $z_{i}$ be the solution of (37)-(38). Then, $z_{i}$ holds the estimate

$$
\|z\|_{\infty} \leq C\|R\|_{\infty}
$$

Proof. The result follows from Lemma 3 taking $A=0$ and $f=R$.

Lemma 5. Let $a, f \in C^{1}(I)$ and $K \in C^{1}(I \times I)$ with

$$
\begin{gathered}
\bar{K}=\max _{I \times I}|K(x, t)|, \\
\left|\frac{\partial}{\partial x} K(x, t)\right| \leq \bar{K}_{1}<\infty,
\end{gathered}
$$

and

$$
\left|\frac{\partial}{\partial t} K(x, t)\right| \leq \bar{K}_{2}<\infty .
$$

Then, the truncation error $R_{i}$ satisfies the estimate

$$
\|R\|_{\infty} \leq C N^{-1} \text {. }
$$

Proof. To establish the estimate given in (43), we proceed by bounding each term in $R_{i}$ provided in (31). For $R_{i}^{(1)}$, we have

$$
\left|R_{i}^{(1)}\right| \leq \chi_{i}^{-1} h_{i}^{-1} \int_{x_{i-1}}^{x_{i}}\left|\left(a^{\prime}(s)\left(x-x_{i}\right)\right) u(x)\right| \varphi_{i}(x) d x
$$

where $s \in\left[x, x_{i}\right]$ comes from the Mean Value Theorem. Then, since $a \in C^{1}(I)$ and from (3) we get

$$
\left|R_{i}^{(1)}\right| \leq C_{1} h_{i}
$$

Further, for $R_{i}^{(2)}$ we take into account of $(40),(41)$ and $\left|T_{0}(\lambda)\right| \leq 1$, so

$$
\begin{aligned}
\left|R_{i}^{(2)}\right| & \leq \lambda \int_{x_{i-1}}^{x_{i}}\left|\left(1+h_{i}^{-1}\left(x-x_{i}\right)\right) \frac{\partial}{\partial \xi}\left(\int_{0}^{\xi} K(\xi, t) u(t) d t\right)\right| d \xi \\
& \leq 2 \lambda \int_{x_{i-1}}^{x_{i}}\left|\frac{\partial}{\partial \xi}\left(\int_{0}^{\xi} K(\xi, t) u(t) d t\right)\right| d \xi .
\end{aligned}
$$

Then, applying the Leibnitz formula to (45) yields

$$
\begin{aligned}
\left|R_{i}^{(2)}\right| & \left.\leq 2 \lambda\left(\int_{x_{i-1}}^{x_{i}}|K(\xi, \xi)||u(\xi)|\right)+\int_{x_{i-1}}^{x_{i}} \int_{0}^{\xi}\left|\frac{\partial}{\partial \xi} K(\xi, t) u(t)\right| d t d \xi\right) \\
& \leq 2 \lambda\left(C \bar{K}+C \bar{K}_{1}\right) h_{i} \\
& \leq C_{2} h_{i} .
\end{aligned}
$$


On the other hand, by the Leibnitz formula and from (40), (42) and (5) we have

$$
\begin{aligned}
\left|R_{i}^{(3)}\right| & \leq \lambda \sum_{j=1}^{i} \int_{x_{j-1}}^{x_{j}}\left(\left|\frac{\partial}{\partial \xi} K\left(x_{i}, \xi\right) u(\xi)\right|+\left|K\left(x_{i}, \xi\right) u^{\prime}(\xi)\right|\right) d \xi \\
& \leq \lambda \sum_{j=1}^{i}\left(C \bar{K}_{2}+\bar{K} \int_{x_{j-1}}^{x_{j}}\left(1+\frac{1}{\varepsilon} e^{-\frac{\alpha \xi}{\varepsilon}}\right)\right) d \xi \\
& =\lambda \sum_{j=1}^{i}\left(C \bar{K}_{2} h_{j}+\bar{K} h_{j}+\alpha^{-1} \bar{K}\left(e^{-\frac{\alpha x_{j-1}}{\varepsilon}}-e^{-\frac{\alpha x_{j}}{\varepsilon}}\right)\right) .
\end{aligned}
$$

Then, by the Mean Value Theorem applied to the exponential term in (47) with $s \in\left[x_{j-1}, x_{j}\right]$ it follows that

$$
\begin{aligned}
\left|R_{i}^{(3)}\right| & \leq \lambda \sum_{j=1}^{i}\left(C \bar{K}_{2} h_{j}+\bar{K} h_{j}+\alpha^{-1} \bar{K} h_{j} e^{-\frac{\alpha s}{\varepsilon}}\right) \\
& \leq C_{3}^{*} i\left|h^{*}\right|,
\end{aligned}
$$

where $h^{*}=\max _{1 \leq j \leq i} h_{j}$. Lastly, for $R_{i}^{(4)}$, similarly to the work above and since $f \in$ $C^{1}(I)$ we have

$$
\begin{aligned}
\left|R_{i}^{(4)}\right| & \leq \chi_{i}^{-1} h_{i}^{-1} \int_{x_{i-1}}^{x_{i}}\left|f^{\prime}(s)\left(x-x_{i}\right)\right| \varphi_{i}(x) d x \\
& \leq C_{4} h_{i}
\end{aligned}
$$

where $s \in\left[x_{i-1}, x_{i}\right]$ by the Mean Value Theorem.

Further in the proof, we need to evaluate each estimate above on the sub-intervals $[0, \sigma]$ and $[\sigma, \ell]$. For this, we first establish the bounds on the step-size $h_{i}$ on each interval. In the first sub-interval $[0, \sigma]$ with $\sigma \leq \frac{\ell}{2}$,

$$
x_{i}=-\alpha^{-1} \varepsilon \ln \left[1-2\left(1-N^{-1}\right) \frac{i}{N}\right], \quad i=1, \cdots, N / 2
$$

and hence,

$$
h_{i}=-\alpha^{-1} \varepsilon \ln \left[1-2\left(1-N^{-1}\right) \frac{i}{N}\right]+\alpha^{-1} \varepsilon \ln \left[1-2\left(1-N^{-1}\right) \frac{i-1}{N}\right] .
$$

Then, we apply the Mean Value Theorem to $h_{i}$ with $i_{*} \in[i-1, i]$ and get

$$
h_{i} \leq \alpha^{-1} \varepsilon \frac{2\left(1-N^{-1}\right) N^{-1}}{1-2 i_{*}\left(1-N^{-1}\right) N^{-1}} \leq C N^{-1} .
$$

In the second sub-interval $[\sigma, \ell]$, we have

$$
x_{i}=\sigma+\left(i-\frac{N}{2}\right) h, \quad i=N / 2+1, \cdots, N,
$$


where $\sigma \leq \frac{\ell}{2}$ and

$$
h_{i}=\frac{2(\ell-\sigma)}{N} \leq C N^{-1} .
$$

Inserting the bounds (50) and (51) in (44), (46), (48) and (49), we have

$$
\left|R_{i}^{(k)}\right| \leq C N^{-1}, \quad k=1,2,3,4 .
$$

which implies the desired result (43).

Theorem 1. Let $u$ be the exact solution of (1)-(2) and $y$ be the solution of (32)(33). If the assumptions on the functions $a, f$ and $K$ from Lemma 5 hold, then

$$
\|y-u\|_{\infty} \leq C N^{-1} .
$$

Proof. The proof follows from Lemma 4 and Lemma 5.

\section{Algorithm and Numerical Results}

In this section, we present the numerical results on an example with an exact solution and an example with an unknown solution. The results include graphs of the approximate solutions, error estimates and the convergence values of the approximate solution to the exact solution. In our algorithm, we consider the following elimination method

$$
\begin{aligned}
y_{i}^{(n)} & =\frac{1}{\varepsilon \theta_{i}+h_{i} a_{i}}\left[\varepsilon \theta_{i} y_{i-1}^{(n}+h_{i}\left(f_{i}-\lambda \sum_{j=1}^{i} h_{j} K\left(x_{i}, x_{j}\right) y_{j}^{(n-1)}\right)\right], \\
y_{0}^{(n)} & =A \\
y_{i}^{(0)} & =A .
\end{aligned}
$$

where $y_{i}^{(0)}$ is the initial process.

Example 1. We study the following initial value problem

$$
\begin{aligned}
\varepsilon u^{\prime}(x)+u(x)+\int_{0}^{x} x u(t) d t= & 2 \varepsilon(x-1)+(x-1)^{2}-\varepsilon x e^{-\frac{x}{\varepsilon}}+\frac{x(x-1)^{3}}{3} \\
& +(\varepsilon-1+x) e^{-x}+\left(\varepsilon-\frac{2}{3}\right) x, \quad 0 \leq x \leq 2, \\
u(0)=1 . &
\end{aligned}
$$

The exact solution of this problem is

$$
u(x)=e^{-\frac{x}{\varepsilon}}+(x-1)^{2}-e^{-x} .
$$

The exact error is calculated by the formula

$$
e_{\varepsilon}^{N}=\left\|y^{N}-u\right\|_{\infty},
$$




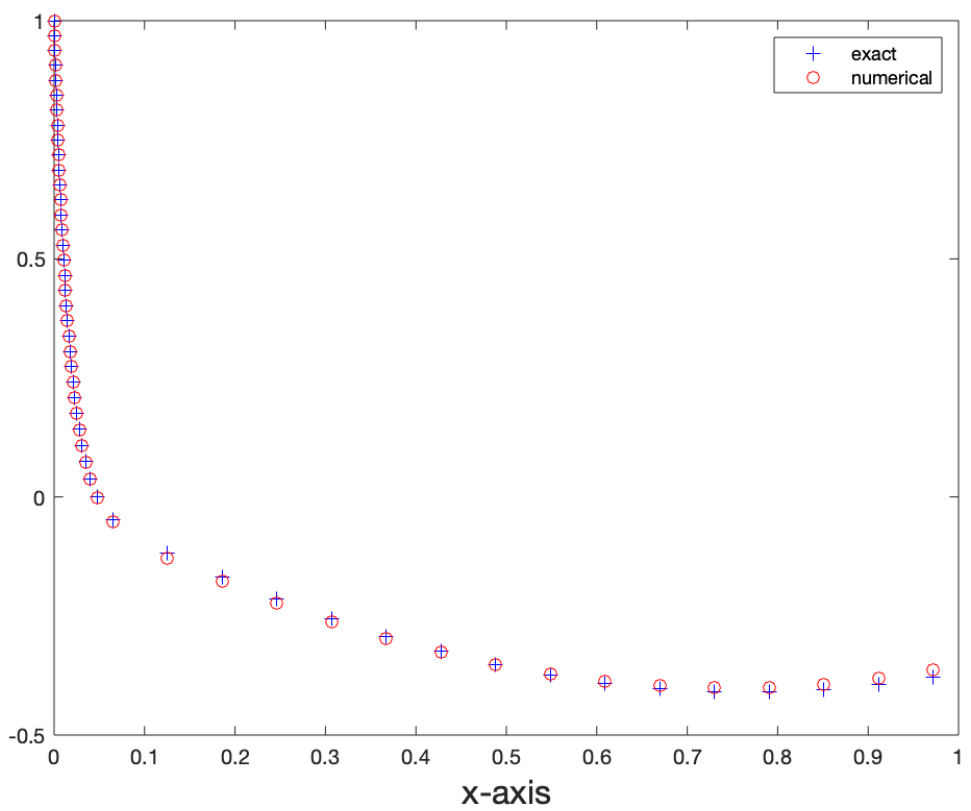

Figure 1. The figure depicts the graphs for the exact solution and the approximate solution for $N=32$.

where $y^{N}$ is the numerical approximation of $u$ for different $N$ and $\varepsilon$ values. We compute the convergence rate by

$$
r^{N}=\frac{\ln \left(e^{N} / e^{2 N}\right)}{\ln 2} .
$$

In Table 1 , we provide the errors $e^{N}, e^{2 N}$ and the convergence rates of the approximate solution for various $N$ and $\varepsilon=2^{-i}$ values.

Example 2. Consider the following test problem

$$
\begin{aligned}
& \varepsilon u^{\prime}+(x+1) u+\int_{0}^{x} x t(x-t)^{2} u(t) d t=x-e^{2 x}, \quad 0 \leq x \leq 2, \\
& u(0)=1 .
\end{aligned}
$$

The exact solution to this problem is not known. To compute the approximate solution and estimate the errors, we utilize the double mesh principle, that is calculating the error of the approximate solution on mesh size $N$ with the approximate solution 
TABLE 1. Errors $e^{N}, e^{2 N}$, and rate of convergence $r$ for Example 1 .

\begin{tabular}{cclllll}
\hline$\varepsilon$ & & $N=32$ & $N=64$ & $N=128$ & $N=256$ & $N=512$ \\
\hline & $e^{N}$ & 0.0651812 & 0.0367749 & 0.0187183 & 0.0091870 & 0.0043264 \\
$2^{-12}$ & $e^{2 N}$ & 0.0298620 & 0.0169626 & 0.0087915 & 0.0042539 & 0.0018714 \\
& $r$ & 1.1261453 & 1.1163687 & 1.0902685 & 1.1104752 & \\
\hline & $e^{N}$ & 0.0653743 & 0.0369845 & 0.0189267 & 0.0093932 & 0.0045311 \\
$2^{-18}$ & $e^{2 N}$ & 0.0300788 & 0.0171765 & 0.0090015 & 0.0044620 & 0.0020767 \\
& $r$ & 1.119980 & 1.1064855 & 1.0721893 & 1.0739417 & \\
\hline & $e^{N}$ & 0.0653777 & 0.0369878 & 0.0189299 & 0.0093964 & 0.0045343 \\
$2^{-24}$ & $e^{2 N}$ & 0.0300821 & 0.0171798 & 0.0090048 & 0.0044652 & 0.0020800 \\
& $r$ & 1.1198837 & 1.1063324 & 1.0719116 & 1.0733905 & \\
\hline
\end{tabular}

computed on double mesh $2 N$, namely

$$
e_{\varepsilon}^{N}=\left\|y^{N}-y^{2 N}\right\|_{\infty}
$$

where $y^{N}$ is the approximate solution on mesh $N$ and $y^{2 N}$ is the approximate solution on mesh $2 N$. The convergence rate is calculated as it is in Example 1.

In Table 2, the errors and the convergence rates of the approximate solution for various $N$ and $\varepsilon=2^{-i}$ values are presented.

TABLE 2. Errors $e^{N}, e^{2 N}$, and rate of convergence $r$ for Example 2 .

\begin{tabular}{lllllll}
\hline$\varepsilon$ & & $N=32$ & $N=64$ & $N=128$ & $N=256$ & $N=512$ \\
\hline \multirow{2}{*}{$2^{-12}$} & $e^{N}$ & 0.0312184 & 0.0156012 & 0.0077960 & 0.0038955 & 0.0019466 \\
& $e^{2 N}$ & 0.0156012 & 0.0077960 & 0.0038955 & 0.0019466 & 0.0009729 \\
& $r$ & 1.0007417 & 1.0008583 & 1.0009223 & 1.0008653 & \\
\hline \multirow{2}{*}{$2^{-18}$} & $e^{N}$ & 0.0312495 & 0.0156246 & 0.0078122 & 0.0039061 & 0.0019530 \\
& $e^{2 N}$ & 0.0156246 & 0.0078122 & 0.0039061 & 0.0019530 & 0.0004882 \\
& $r$ & 1.0000121 & 1.0000146 & 1.0000172 & 1.0000198 & \\
\hline & $e^{-24}$ & 0.0312500 & 0.0156250 & 0.0078125 & 0.0039063 & 0.0019531 \\
& $e^{2 N}$ & 0.0156250 & 0.0078125 & 0.0039063 & 0.0019531 & 0.0009766 \\
& $r$ & 1.0000002 & 1.0000002 & 1.0000003 & 1.0000003 & \\
\hline
\end{tabular}

\section{Conclusion}

To sum up, we constructed a finite difference scheme on a Bakhvalov-Shishkin mesh to obtain the numerical solution of an initial value problem for a linear firstorder singularly perturbed Volterra integro-differential equation with a boundary layer. We proved that the method is first-order uniformly convergent with respect to the perturbation parameter. As we can see in Table 1, Table 2 and Figure 1, the numerical results of the test problems are also consistent with the analysis on the error estimates and convergence order and hence, it is confirmed that the convergence order of the scheme $O\left(N^{-1}\right)$. For future work, we suggest that this difference scheme method on Bakhvalov-Shishkin mesh can be applied to the singularly perturbed linear or non-linear problems with delay to obtain accurate numerical solutions. Further, our proposed scheme can be modified to handle integro-differential 
equations with fractal derivatives which are studied in 8 .

Author Contribution Statements All authors contributed equally to this work, and they read and approved the final manuscript.

Declaration of Competing Interests The authors declare that they have no known competing financial interest or personal relationships that could have appeared to influence the work reported in this paper.

Acknowledgement The authors are thankful to the referee for making valuable suggestions leading to the better presentations of the paper.

\section{REFERENCES}

[1] Amiraliyev, G. M., Mamedov, Y. D. H., Difference schemes on the uniform mesh for singular perturbed pseudo-parabolic equations, Turk. J. Math., 19 (1995), 207-222.

[2] Amiraliyev, G. M., Sevgin, S., Uniform difference method for singularly perturbed Volterra integro-differential equations, Appl. Math. Comput., 179 (2006), 731-741, https://dx.doi.org/10.1016/j.amc.2005.11.155.

[3] Amiraliyev, G. M., Yapman, O., A novel second-order fitted computational method for a singularly perturbed Volterra integro-differential equation, International Journal of Computer Mathematics, 97-6 (2020), 1293-1302, https://dx.doi.org/10.1080/00207160.2019.1614565.

[4] Amiraliyev, G. M., Yilmaz, B., Fitted difference method for a singularly perturbed initial value problem, Int. J. Math. Comput., 22 (2014), 1-10.

[5] Angell, J. S., Olmstead, W. E., Singular perturbation analysis of an integro-differential equation modelling filament stretching, J. Appl. Math. Phys. (ZAMP), 36 (1985), 487-490, https://dx.doi.org/10.1007/BF00944639.

[6] Angell, J. S., Olmstead, W. E., Singularly perturbed Volterra integral equations, SIAM J. Appl. Math., 47 (1987), 1150-1162, https://dx.doi.org/10.1137/0147001.

[7] Angell, J. S., Olmstead, W. E., Singularly perturbed Volterra integral equations ii, SIAM J. Appl. Math., 47 (1987), 1-14, https://dx.doi.org/10.1137/0147077.

[8] Anjum, N., He, C. H., He, J. H., Two-scale fractal theory for the population dynamics, Fractals, https://dx.doi.org/10.1142/S0218348X21501826.

[9] Anjum, N., He, J.-H., Homotopy perturbation method for N/MEMS oscillators, Math Meth Appl Sci., 1-15, https://dx.doi.org/10.1002/mma.6583.

[10] Arslan, D., A uniformly convergent numerical study on Bakhvalov-Shishkin mesh for singularly perturbed problem, Communications in Mathematics and Applications, 11-1 (2020), 161-171.

[11] Cakir, M., Gunes, B., Duru, H., A novel computational method for solving nonlinear Volterra integro-differential equation, Kuwait J. Sci, 48 (1) (2021), 1-9, https://dx.doi.org/10.48129/kjs.v48i1.9386.

[12] Cen, Z., Le, A., Xu, A., Parameter-uniform hybrid difference scheme for solutions and derivatives in singularly perturbed initial value problems, J. Comput. Appl. Math., 320 (2017), 176-192, https://dx.doi.org/10.1016/j.cam.2017.02.009.

[13] Cushing, J. M., Integro-differential Equations and Delay Models in Population dynamics, Springer-Verlag, New York, 1992.

[14] De Gaetano, A., Arino, O., Mathematical modelling of the intravenous glucose tolerance test, J. Math. Biol., 40 (2000), 136-168, https://dx.doi.org/10.1007/s002850050007. 
[15] Doolan, E. P., Miller, J. J. H., Schilders, H. A., Uniform Numerical Method for Problems with Initial and Boundary Layers, Boole Press, Dublin, 1980.

[16] Farrel, P. A., Hegarty, A. F., Miller, J. J. H., O'Riordan, E., Shishkin, G. I., Robust Computational Techniques for Boundary Layers, Chapman Hall/CRC, New York, 2000, https://dx.doi.org/10.1201/9781482285727.

[17] He, J., El-Dib, Y. O., Homotopy perturbation method for Fangzhu oscillator, J. of Math. Chem., 58 (2020), 2245-2253, https://dx.doi.org/10.1007/s10910-020-01167-6.

[18] He, J.-H., A simple approach to Volterra-Fredholm integral equations, Journal of Applied and Computational Mechanics, 6 (Special Issue) (2020), 1184-1186, https://dx.doi.org/10.22055/jacm.2020.34653.2451.

[19] Horvat, V., Rogina, M., Tension spline collocation methods for singularly perturbed Volterra integro-differential and Volterra integral equations, J. Comput. Appl. Math., 140 (7) (2002), 381-402.

[20] Jordan, G. . S., Some nonlinear singularly perturbed Volterra integro-differential equations, in: Volterra Equations (Proc. Helsinki Sympos. Integral Equations, Otaniemi, 1978), Lecture Notes in Mathematics, 737 (1979), 107-119, https://dx.doi.org/10.1007/BFb0064501.

[21] Jordan, G. S., A nonlinear singularly perturbed Volterra integro-differential equation of nonconvolution type, Proc. Roy. Soc. Edinburgh Sect. A, 80 (1978), 235-247, https://dx.doi.org/10.1017/S030821050001026X.

[22] Kadalbajoo, M. K., Gupta, V., A brief survey on numerical methods for solving singularly perturbed problems, Appl. Math. Comput., 217 (2010), 3641-3716, https://dx.doi.org/10.1016/j.amc.2010.09.059.

[23] Kauthen, J. P., Implicit Runge-Kutta methods for some integro-differential-algebraic equations, Appl. Numer. Math., 13 (1993), 125-134.

[24] Kauthen, J. P., Implicit Runge-Kutta methods for singularly perturbed integro-differential systems, Appl. Numer. Math., 18 (1995), 201-210, https://dx.doi.org/https://doi.org/10.1016/0168-9274(95)00053-W.

[25] Kauthen, J. P., A survey of singularly perturbed Volterra equations, Appl.Numer.Math, 24 (1997), 95-114.

[26] Khater, A. H., Shamardan, A. B., Callebaut, D. K. Sakran, M. R. A., Numerical solutions of integral and integro- differential equations using Legendre polynomials, Numer. Algorithms, 46 (2007), 195-218, https://dx.doi.org/10.1007/s11075-007-9130-2.

[27] Kudu, M., Amirali, I., Amiraliyev, G. M., A finite-difference method for a singularly perturbed delay integro-differential equation, J. Comput. Appl. Math., 308 (2016), 379-390, https://dx.doi.org/10.1016/j.cam.2016.06.018.

[28] Kumar, M., Singh, P., Misra, H. K., A recent survey on computational techniques for solving singularly perturbed boundary value problems, Int. J. Comput. Math., 84(10) (2007), 14391463, https://dx.doi.org/10.1080/00207160701295712.

[29] Kumar, V., Srinivasan, B., An adaptive mesh strategy for singularly perturbed convection diffusion problems, Applied Mathematical Modelling,, 39 (7) (2015), 2081-2091, https://dx.doi.org/10.1016/j.apm.2014.10.019.

[30] Linss, T., Analysis of a Galerkin finite element method on a Bakhvalov-Shishkin mesh for a linear convection-diffusion problem, IMA Journal of Numerical Analysis, 20 (2000), 621-632, https://dx.doi.org/10.1093/imanum/20.4.621.

[31] Linss, T., Layer-adapted meshes for convection-diffusion problems, Computer Methods in Applied Mechanics and Engineering, 192 (9-10) (2003), 1061-1105, https://dx.doi.org/10.1007/978-3-642-05134-0.

[32] Lodge, A. S., McLeod, J. B., Nohel, J. A., A nonlinear singularly perturbed Volterra integrodifferential equation occurring in polymer rheology, Proc. Roy. Soc. Edinburgh Sect. A, 80 (1978), 99-137, https://dx.doi.org/10.1017/S0308210500010167. 
[33] Marino, S., Beretta, E., Kirschner, D. E., The role of delays in innate and adaptive immunity to intracellular bacterial infection, Math. Biosci. Eng., 4 (2007), 261-286, https://dx.doi.org/10.3934/mbe.2007.4.261.

[34] Miller, J. J. H., O'Riordan, Shishkin, G. I., Fitted Numerical Methods for Singular Perturbation Problems, Revised Edition, World Scientific, 2012, https://dx.doi.org/10.1142/8410.

[35] Mishra, H. K., S. S., Various numerical methods for singularly perturbed boundary value problems, Am. J. Appl. Math. Statist., 2 (2014), 129-142, https://dx.doi.org/10.12691/ajams-2-3-7.

[36] Ramos, J. I., Exponential techniques and implicit Runge-Kutta method for singularlyperturbed Volterra integro- differential equations, Neural Parallel Sci. Comput., 16 (2008), 387-404.

[37] Reddingius, J., Notes on the mathematical theory of epidemics, Acta Biotheor., 20 (1971), 125-135, https://dx.doi.org/10.1007/BF01556687.

[38] Roos, H. G., Stynes, M., Tobiska, L., Numerical Methods for Singularly Perturbed Differential Equations, Springer- Verlag, Berlin, 1996, https://dx.doi.org/10.1007/978-3-662-03206-0.

[39] Salama, A. A., Bakr, S. A., Difference schemes of exponential type for singularly perturbed Volterra integro-differential problems, Appl. Math. Model., 31 (2007), 866-879, https://dx.doi.org/10.1016/j.apm.2006.02.007.

[40] Wazwaz, A., Linear and Nonlinear Integral Equations, Springer, 2011, https://dx.doi.org/10.1007/978-3-642-21449-3. 